\title{
Mycobacterium saskatchewanense sp. nov., a novel slowly growing scotochromogenic species from human clinical isolates related to Mycobacterium interjectum and Accuprobe- positive for Mycobacterium avium complex
}

\author{
Correspondence \\ C. Y. Turenne \\ cturenne@hc-sc.gc.ca
}

\author{
C. Y. Turenne, ${ }^{1}$ L. Thibert, ${ }^{2}$ K. Williams, ${ }^{3}$ T. V. Burdz, ${ }^{1}$ V. J. Cook, ${ }^{4}$ \\ J. N. Wolfe, ${ }^{1}$ D. W. Cockcroft ${ }^{3}$ and A. Kabani ${ }^{1,5}$ \\ ${ }^{1}$ National Reference Centre for Mycobacteriology, National Microbiology Laboratory, Health \\ Canada, Winnipeg, Manitoba, Canada R3E 3R2 \\ ${ }^{2}$ Laboratoire de santé publique du Québec, Institut national de santé publique du Québec, \\ Sainte-Anne-de-Bellevue, Québec, Canada \\ ${ }^{3}$ Royal University Hospital, Saskatoon, Saskatchewan, Canada \\ ${ }^{4,5}$ Division of Respiratory Medicine, Department of Medicine ${ }^{4}$ and Department of Medical \\ Microbiology ${ }^{5}$, University of Manitoba, Winnipeg, Manitoba, Canada
}

\section{INTRODUCTION}

Organisms of the Mycobacterium avium complex (MAC) are the non-tuberculous mycobacteria (NTM) species isolated most often in clinical laboratories (Wallace et al., 1997). MAC organisms are often the cause of chronic pulmonary disease in older individuals, lymphadenitis in children and disseminated disease in immunocompromised patients (Wallace et al., 1997) and their detection and

Published online ahead of print on 31 October 2003 as DOI 10.1099/ ijs.0.02739-0.

Abbreviations: AFB, acid-fast bacillus; ITS, internal transcribed spacer; MAC, M. avium complex; NTM, non-tuberculous mycobacteria; PRA, PCR-restriction enzyme analysis.

The GenBank/EMBL/DDBJ accession numbers for the 16S rRNA gene, ITS1 and partial hsp65 gene sequences of strains $00-250^{\top}$ and MB54784 are AY208856-AY208859.

Details of the strains analysed in this study, photos of colonies and an ITS1 alignment are available as supplementary material in IJSEM Online. identification is crucial to the administration of appropriate antimicrobial therapy. The availability of a commercial nucleic acid probe (AccuProbe; Gen-Probe) for the identification of MAC species has made the speciation process rapid, accurate and easy, particularly in comparison with the tedious and often ambiguous biochemical tests algorithms.

Strains that are not M. avium or Mycobacterium intracellulare but that are MAC DNA probe positive, often referred to as MAIX isolates or ' $\mathrm{X}$ ' strains, have been described previously (Cregan et al., 1992; Smole et al., 2002; Soini et al., 1994, 1996; Tortoli et al., 1997; Viljanen et al., 1993). Sequence-based evaluations of the $32 \mathrm{kDa}$ protein (Soini et al., 1994, 1996), internal transcribed spacer (ITS1) region (Frothingham \& Wilson, 1993) and hsp65 gene (Smole et al., 2002) have suggested that some MAIX strains represent novel species within the complex. We present a clinically significant case of NTM pulmonary infection caused by a novel species of mycobacteria that is MAC AccuProbe positive, but presents with a unique combination of 
genotypic and phenotypic characteristics. Additional strains with identical characteristics were identified from other specimens and have been included in this study.

\section{METHODS}

Case history. The patient is a 72-year-old female retired nurse with a 52-year history of recurrent chest infections. She had been on intermittent antibiotic therapy since her late teens but was never hospitalized until 1990, when she developed a right middle lobe pneumonia with lobar collapse. Persistent infection led to a right middle lobectomy in 1991. Pre-operatively, a computed tomography (CT) scan showed extensive bronchiectasis in the right middle lobe, left lower lobe and lingula. Her sputum was negative for acid-fast bacilli (AFB) at that time and her immunoglobulin and sweat chlorides levels were normal. No obvious associations were made for her bronchiectasis, aside from previous recurrent infections. In 1995, the patient's bronchiectasis showed gradual radiographic progression, though cultures for AFB remained negative. New onset of haemoptysis prompted extensive re-evaluation in July 1999. Progressive bronchiectasis was documented and three sputa specimens were positive for AFB identified as Mycobacterium gordonae by conventional laboratory methodology. No treatment was offered for this commensal organism, although courses of antibiotics were directed against conventional bacterial pathogens and her haemoptysis settled.

Re-evaluation by a respiratory specialist, including chest X-ray and pulmonary function tests, in August 2000 revealed new left upper lobe cavitary lesions. Multiple sputum specimens were positive for AFB $(4+)$, identified by nucleic acid probe as MAC. The patient deteriorated rapidly and was admitted to hospital presenting with a new left pleural effusion and left lower lobe pneumonia. She did not respond to 5 days of intravenous piperacillin/tazobactam and was then placed on a three-drug regimen of ciprofloxacin, azithromycin and amikacin, to which she responded clinically within $48 \mathrm{~h}$. After 5 weeks on this regimen, clinical response was marked and her pleural effusion had resolved, although her sputum samples continued to reveal AFB $(1+)$ with positive culture for M. avium based on nucleic acid probes. After 8 weeks of therapy, rifabutin was substituted for amikacin. Compliance with this new regimen was approximately $60 \%$, due to gastrointestinal intolerance, and rifabutin was discontinued after 4 weeks due to a fourfold rise in her hepatic transaminases. After 5 months on the two-drug regimen (ciprofloxacin, azithromycin), sputum was negative for AFB by stain and culture and she was clinically well with stable X-rays. After a treatment ration of 9 months, sputum cultures remain negative for AFB.

Concurrently, one of the strains isolated in summer 2000, strain $00-250^{\mathrm{T}}$, was submitted to the National Reference Centre for Mycobacteriology for MAC susceptibility testing, where the unusual morphology of the organism prompted 16S rRNA gene sequence identification. It was determined that the organism was distinct from any established mycobacterium species and was more closely related to Mycobacterium interjectum on the basis of $16 \mathrm{~S}$ rDNA analysis. Two other isolates were obtained from the patient and were submitted for $16 \mathrm{~S}$ rDNA sequencing identification. One was from sputum (strain 01-244), which was isolated earlier, in July 1999, and identified by biochemical tests as M. gordonae. The other strain was from thoracenthesis fluid (strain 00-308, isolated September 2000). It was determined that the same organism was isolated in each case.

Strains. Three strains from the patient were made available for this study. One of these, strain $00-250^{\mathrm{T}}$, was sent for HPLC analysis to the Laboratoire de Santé Publique du Québec and showed a similar pattern to several isolates identified by HPLC as M. interjectum-like from various regions of Québec over the last decade. Sequencing of the 16S rDNA indicated that a great proportion of these were identical to the strains isolated from the case discussed above and were included in this study to provide a better representation of the susceptibility and phenotypic and genotypic characteristics of this novel species. Strain isolation and patient data of the 16 strains (13 from Québec and three from the case report) are available as a supplementary table in IJSEM Online.

Phenotypic properties. A standard series of biochemical tests, growth temperatures and morphological examination for mycobacteriology were performed as described previously (Kent \& Kubica, 1985), as listed in Table 1.

Susceptibility testing was performed using serial twofold broth microdilution as described previously (Brown et al., 1992; NCCLS, 2000; Wallace et al., 1986). Microdilution plates were prepared inhouse using Middlebrook 7H9 broth (Difco) supplemented with $5 \%$ oleic acid/albumin/dextrose enrichment. A range of seven or eight concentrations was tested for each antimicrobial. Stock concentrations of the following antimicrobial agents were prepared from manufacturers' powders: rifampicin, ethambutol, isoniazid, amikacin and sulfamethoxazole (Sigma), ciprofloxacin and moxifloxacin (Bayer), rifabutin and linezolid (Pharmacia), streptomycin (ICN), clarithromycin (Abbott) and levofloxacin (Jansen-Othro). The 13 Québec isolates and strain $00-250^{\mathrm{T}}$ from the case history were tested at a final inoculum of $10^{3}-10^{4}$ c.f.u. $\mathrm{ml}^{-1}$. Microdilution plates were sealed in plastic bags and incubated at $37^{\circ} \mathrm{C}$ until macroscopic growth was sufficient for interpretation (7-14 days). MICs were defined as the lowest concentration of drug to inhibit visible growth, with the exception of sulfamethoxazole, for which the MIC is determined at $80 \%$ inhibition of growth compared with the growth control well. Quality control was performed using M. avium ATCC 700898, Mycobacterium marinum ATCC $927^{\mathrm{T}}$ (Wallace et al., 1986) and M. avium NJ-9141 (Heifets et al., 1993). Staphylococcus aureus ATCC 29213 was used as a quality-control strain in cation-supplemented Mueller-Hinton broth when MIC ranges for mycobacteria were not available.

DNA probe. AccuProbe assays (Gen-Probe) for MAC were performed using colonies from pure culture of the 16 isolates in this study.

HPLC. HPLC by UV detection was performed according to the standard methodology (Butler et al., 1996).

Sequencing of the 165 rDNA and ITS1 region. A loopful of organism from pure culture was suspended in sterile distilled water, boiled for $10 \mathrm{~min}$ and then subjected to mechanical lysis using a Mini Bead-Beater (Biospec Products). Lysates were then centrifuged for $2 \mathrm{~min}$ at $12000 \mathrm{~g}$ and the supernatant was used for PCR. Amplification of the 16S rRNA gene (Rogall et al., 1990; Turenne et al., 2001) and the ITS1 region (Harmsen et al., 2002; Turenne et al., 2002) was performed as described previously and PCR products were sequenced directly using forward and reverse primers and loaded on an ABI PRISM 310 Genetic Analyzer capillary sequencer (Applied Biosystems). Sequence editing and phylogenetic analyses by CLUSTAL V (neighbour-joining method) were performed using the SEQMAN and MEGALIGN components of Lasergene 5 (DNASTAR).

Sequencing and PCR-restriction enzyme analysis (PRA) of the hsp65 gene. A 441-bp fragment of the hsp65 gene was amplified from the DNA lysates prepared above using the protocol described previously (Telenti et al., 1993). The resulting PCR product was sequenced using the forward and reverse PCR primers TB11 and TB12. Computer-generated PRA profiles of 441-bp sequences with enzymes HaeII and BstEII were determined using MapDraw (DNASTAR). 
Table 1. Phenotypic characteristics of the study strains in comparison with other slowly growing Mycobacterium species

Strains/species: 1, study strains $(n=15) ; 2$, strain MB54784; 3, strain MCRO 8 (Springer et al., 1996); 4. M. palustre (Torkko et al., 2002); 5. M. interjectum 4185/92 ${ }^{\mathrm{T}}$ (Springer et al., 1993; Tortoli et al., 1996); 6, M. avium complex; 7, M. gordonae (Kent \& Kubica, 1985). Percentages of strains giving the indicated test result are given in parentheses. All species or strains grew at $25-37^{\circ} \mathrm{C}$ and were negative for niacin and tolerance of $5 \% \mathrm{NaCl}$ (not determined for $M$. palustre). ND, No data; $\mathrm{V}$, variable; $\mathrm{W}+$, weakly positive.

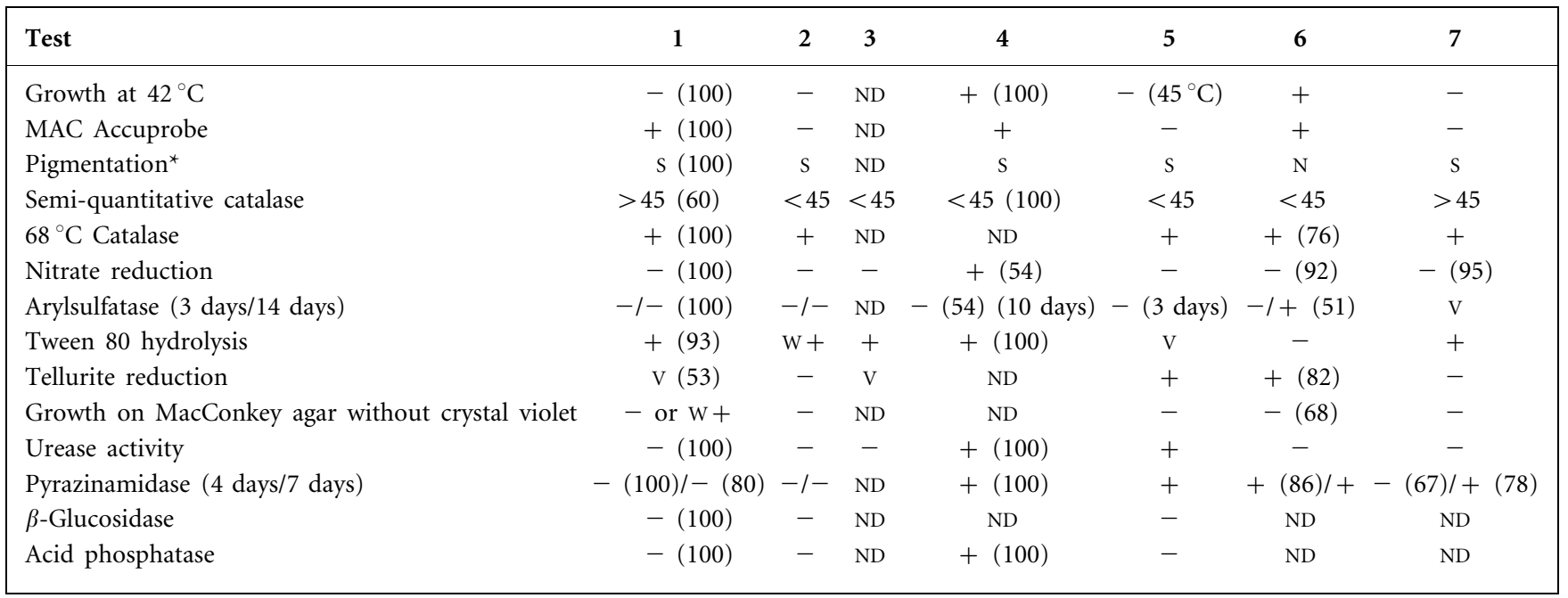

${ }^{\star} \mathrm{N}$, Non-chromogen; S, scotochromogen.

\section{RESULTS AND DISCUSSION}

\section{Phenotypic properties and AccuProbe}

Acid-fast microscopy revealed beaded and dispersed forms of AFB. Growth occurred best at $37^{\circ} \mathrm{C}$ with colonies appearing after 2-4 weeks. On Middlebrook 7H10 solid medium, the scotochromogenic strains were initially transparent in the first 2 weeks of growth, with mature colonies showing a bright yellow pigment, most with a raised centre and a transparent, irregular apron. Photos of colonies on Middlebrook 7H10 agar are available as Supplementary Fig. A in IJSEM Online. Growth on Lowenstein-Jensen solid medium was rough and dry in texture. Strain MB54784 showed domed, smooth colonies. Biochemical test results and growth temperatures determined for each strain are indicated in Table 1. All strains, with the exception of strain MB54784 with an RLU of 1142, were positive for the MAC AccuProbe assay.

\section{Susceptibility testing}

According to current NCCLS guidelines for susceptibility testing of MAC and proposed resistance breakpoints for Mycobacterium kansasii (NCCLS, 2000), all isolates were resistant to isoniazid and sensitive to rifampicin, rifabutin, streptomycin, clarithromycin, amikacin, ciprofloxacin and sulfamethoxazole (Table 2). For ethambutol, $86 \%$ of isolates were susceptible using a resistance breakpoint of $10 \mu \mathrm{g} \mathrm{ml}^{-1}$. If proposed breakpoints for linezolid and rapidly growing mycobacteria are employed (Wallace et al., 2001), all isolates were susceptible to this antimicrobial.
Low MIC values for moxifloxacin and levofloxacin may indicate in vitro susceptibility to these flouroquinolones.

\section{Mycolic acid pattern by HPLC}

With minor peak differences not affecting the overall appearance, the distinctive nine-peak pattern produced by $00-250^{\mathrm{T}}$, as well as of all the other isolates, belongs to the group of late-emerging, single-cluster peak patterns, such as those of M. kansasii and Mycobacterium kubicae (Fig. 1), but with more and better-developed late peaks. It is almost indistinguishable from that of the newly described $\mathrm{Myco}$ bacterium palustre, also an MAC AccuProbe-positive species (Torkko et al., 2002). With its central double peak and higher end peaks, it is very similar to some published profiles of strains as well as of a Québec clinical single cluster pattern identified as M. interjectum (Lumb et al., 1997), distinct from the double-cluster pattern of the type strain of M. interjectum (Butler \& Guthertz, 2001).

\section{Sequencing of the 16S rRNA gene}

The nearly complete $16 \mathrm{~S}$ rRNA gene sequence, corresponding to positions 28-1483 of the Escherichia coli sequence, was determined for strain $00-250^{\mathrm{T}}$ as well as the $5^{\prime}$ region, positions 54-510, of all subsequent isolates. All isolates were identical to $00-250^{\mathrm{T}}$ in the $5^{\prime}$ region, with the exception of a 1-bp mismatch in isolate MB54784. Sequencing of the rest of the $16 \mathrm{~S} \mathrm{rDNA}$ for this isolate revealed that no other mismatches from $00-250^{\mathrm{T}}$ were present. The sequence contains the characteristic 12-bp omission (short helix) in the hypervariable region 18 (V3) observed in rapidly 
Table 2. Antimicrobial sensitivity results of 14 strains as determined by the broth microdilution method

MIC values may be less than or equal to the concentration indicated.

\begin{tabular}{|c|c|c|c|c|c|c|c|c|c|c|c|c|}
\hline \multirow[t]{2}{*}{ Antimicrobial } & \multirow{2}{*}{$\begin{array}{l}\text { Concentration range } \\
\text { tested }\left(\mu \mathrm{g} \mathrm{ml}^{-1}\right)\end{array}$} & \multicolumn{11}{|c|}{ Cumulative percentage of isolates for which MIC is $\left(\mu \mathrm{g} \mathrm{ml}^{-1}\right)$ : } \\
\hline & & $0 \cdot 03$ & $0 \cdot 06$ & $0 \cdot 125$ & $0 \cdot 25$ & $0 \cdot 5$ & 1 & 2 & 4 & 8 & 16 & $\geqslant 32$ \\
\hline Amikacin & $0 \cdot 63-80 \cdot 0$ & & & & & & 14 & 64 & 100 & & & \\
\hline Ciprofloxacin & $0 \cdot 125-16 \cdot 0$ & & & 50 & 79 & 100 & & & & & & \\
\hline Clarithromycin & $1 \cdot 0-128 \cdot 0$ & & & & & & 100 & & & & & \\
\hline Ethambutol & $0 \cdot 31-40 \cdot 0$ & & & & & & 7 & 21 & 64 & 86 & 100 & \\
\hline Isoniazid & $0 \cdot 31-40 \cdot 0$ & & & & & & & & & & & 100 \\
\hline Levofloxacin & $0 \cdot 03-4 \cdot 0$ & 14 & & 29 & 71 & 100 & & & & & & \\
\hline Linezolid & $0 \cdot 5-64 \cdot 0$ & & & & & 21 & 79 & 100 & & & & \\
\hline Moxifloxacin & $0 \cdot 03-4 \cdot 0$ & 86 & & & 93 & 100 & & & & & & \\
\hline Rifabutin & $0 \cdot 03-4 \cdot 0$ & 100 & & & & & & & & & & \\
\hline Rifampicin & $0 \cdot 06-8 \cdot 0$ & & 100 & & & & & & & & & \\
\hline Streptomycin & $0 \cdot 63-80 \cdot 0$ & & & & & 64 & 71 & 86 & 100 & & & \\
\hline Sulfamethoxazole & $2 \cdot 0-256 \cdot 0$ & & & & & & & & 21 & 64 & 93 & 100 \\
\hline
\end{tabular}

growing mycobacteria, Mycobacterium simiae and other slowly growing species related to $M$. simiae. Its closest matches using the RIDOM database (Harmsen et al., 2002) were Mycobacterium lentiflavum ATCC 51988 and DSM
$44418^{\mathrm{T}}$ (=ATCC $51985^{\mathrm{T}}$ ), with 5 base mismatches in the region analysed (E. coli positions 54-510). Analysis of the nearly complete $16 \mathrm{~S}$ rDNA using BLAST against sequences from GenBank, EMBL or DDBJ revealed an identical match
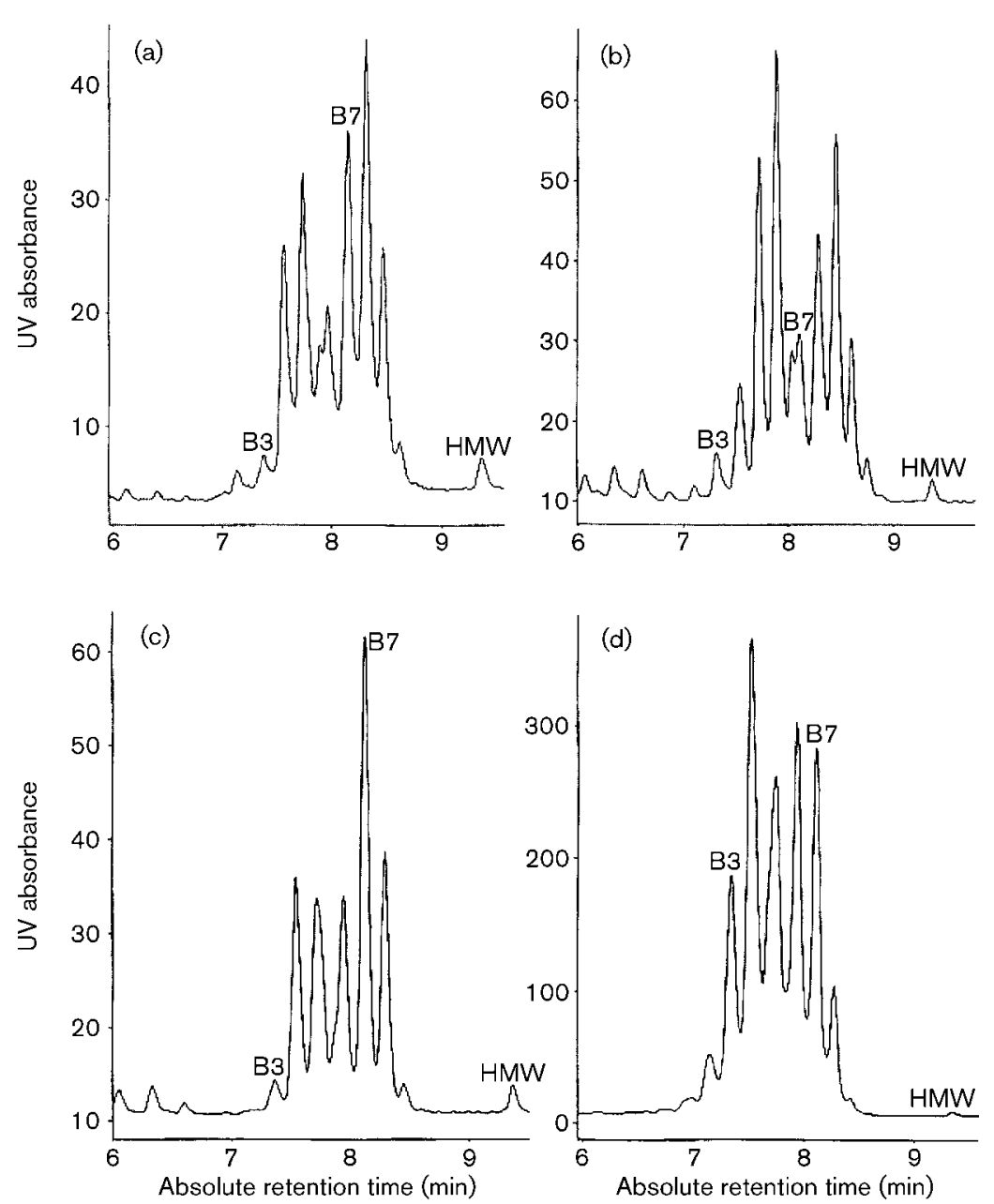

Fig. 1. Mycolic acid patterns of strain 00$250^{\top}$ (a), a clinical strain of $M$. interjectum subspecies MB51494 (b), M. kubicae ATCC $700732^{\top}$ (c) and M. kansasii ATCC $12478^{\top}$ (d) obtained by HPLC analysis. Peak designation is as previously suggested. HMW: High molecular mass internal standard. Strain MB51494 was identified by $16 \mathrm{~S}$ rRNA gene sequence analysis as M. interjectum Patient 2 (GenBank accession no. AF014936) (Lumb et al., 1997). 
with Mycobacterium sp. MCRO 8 (X93034), with the exception of two Ns in the MCRO 8 sequence. MCRO 8 has been described as a unique species most closely resembling Mycobacterium scrofulaceum biochemically and was presumptively identified as a 'slowly growing species related to M. simiae' by $16 \mathrm{~S}$ rDNA analysis (Springer et al., 1996). Final sequence analysis was carried out using our in-house sequence database (in combination with GenBank sequences) and evaluating positions $28-1483$ on the E. coli $16 \mathrm{~S}$ rRNA gene (Fig. 2). Using the type strains of established species, the closest relatives were $M$. interjectum (similarity $99 \cdot 1 \%$; 13 differences), Mycobacterium heidelbergense (98.8\%; 17), Mycobacterium lentiflavum $(98.6 \% ; 19)$, M. simiae $(98 \cdot 3 \% ; 23)$ and Mycobacterium intermedium $(98 \cdot 3 \% ; 24)$. An alignment of hypervariable regions $A$ and $B$ revealed signature sequences in the A region (Fig. $3 \mathrm{a}$ ), with the B region being identical to that of $M$. simiae.

\section{Sequencing of the ITS1}

All strains revealed identical ITS1 sequences, with the exception of strain MB54784, which differed from the consensus sequence of all other strains by a hamming distance of 7 . Neither of the sequences corresponded to ITS1 sequences in public databases. BLAST search analysis revealed closest similarity to Mycobacterium malmoense (AF367025), with $91 \%$ identity in a partial overlap of 335 bases, while RIDOM analysis indicated closest similarity to $M$. intracellulare S348, with $87 \cdot 54 \%$ identity in an overlap of only 281 bases.

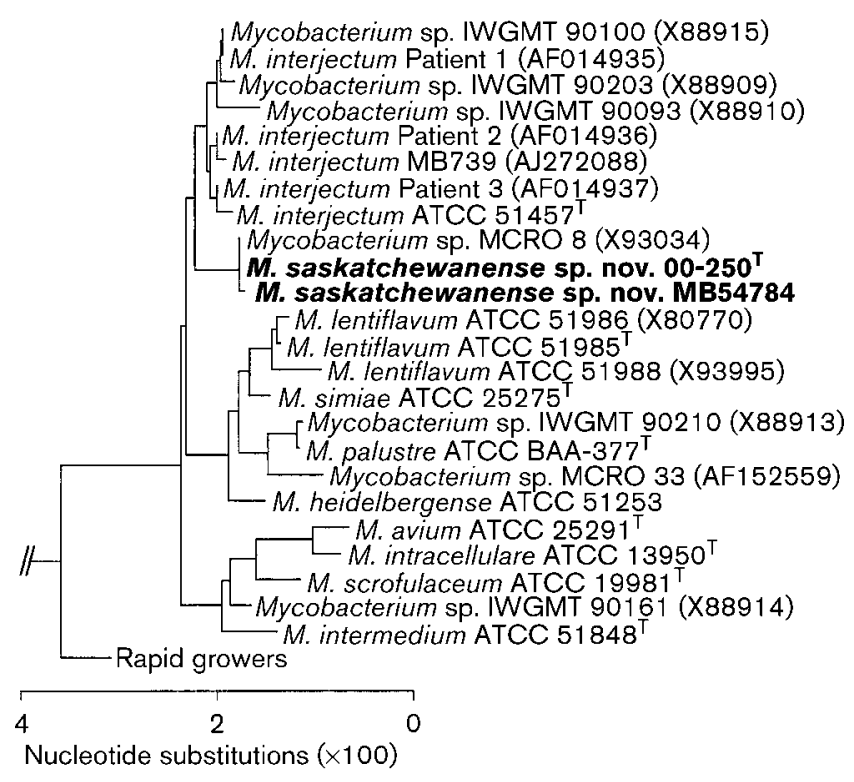

Fig. 2. CLUSTAL $W$ analysis of strain $00-250^{\top}$ with its closest genotypic relatives. End gaps were removed to include positions 54-1470 of the $E$. coli 16S rRNA gene, with the exception of accession numbers AF014935, AF014936 and AF014937, which begin at position 110. Sequences were determined in our laboratory unless indicated by accession numbers in parentheses.
The ITS1 sequence of $M$. interjectum ATCC $51457^{\mathrm{T}}$ revealed $83 \cdot 1$ and $81 \cdot 0 \%$ identity against strains $00-250^{\mathrm{T}}$ and MB54784, respectively, thereby confirming its distinction from them. An ITS1 alignment is available as Supplementary Fig. B in IJSEM Online.

\section{Sequencing and PRA of the hsp65 gene}

Sequences of a 441-bp region of $h s p 65$ revealed four distinct sequevars, Msk1, Msk2, Msk3 and Msk4 (Supplementary Table, Fig. 3b), most with 1 or 2 base variations from each other. The exception was strain MB54784 from Québec, or sequevar Msk4, which differed by 20 bases from Msk1. Msk1 was the most prevalent, followed by sequevar Msk2, which differed from Msk1 by 1 base, at position 411 of the 441-bp fragment. Sequevar Msk3, represented by strain MB13405 alone, differed from Msk1 by 2 bases, at positions 264 and 411 of the 441-bp fragment. The closest established species as determined by $16 \mathrm{~S}$ rDNA analysis were also sequenced for the partial hsp 65 gene and compared with the clinical isolates (Fig. 3b). Sequevars Msk1, Msk2 and Msk3 are most closely related to $M$. interjectum ATCC $51457^{\mathrm{T}}$, differing from Msk 1 by 7 bases. BLAST analysis of the partial hsp65 sequence was performed with Msk1 and Msk4, using positions 46-405 of the 441-bp fragment to obtain the most accurate top score with mycobacterial hsp65 sequences in the public database, most being either 401 or $360 \mathrm{bp}$ in length. BLAST results of Msk1 revealed a top score with $M$. interjectum (AJ307632), with seven mismatches, corresponding to that determined with our in-house sequence of the type strain. BLAST results of strain MB54784 (Msk4) revealed a top score and eight mismatches with M. gordonae SN 601 (AF434735), which differs from M. gordonae ATCC $14470^{\mathrm{T}}$ (AF434734) by 30 bases.

Computerized restriction maps of the 441-bp hsp65 fragment as determined by MAPDRAW (LaserGene) revealed the presence of fragments of 231 and 210 bp with BstEII and 127 and 145 bp with HaeIII digestion in sequevars Msk1, Msk2 and Msk3 (Table 3). Fragments smaller than 50 bp were not taken into account. This pattern corresponds exactly with that of M. interjectum (da Silva Rocha et al., 2002), M. avium III (Leao et al., 1999) and MAC isolate Mav v3 (Smole et al., 2002). Sequevar Msk4, however, presents a PRA pattern of 231, 131 and 79 bp fragments with BstEII and 127 and $94 \mathrm{bp}$ fragments with HaeIII, which does not correspond to published PRA patterns derived from sequence analysis (Brunello et al., 2001).

\section{Comparison with possibly similar strains in the literature}

Strains were not tested using the individual M. avium or $M$. intracellulare probes, though, with the exception of strain MB54784, all were MAC AccuProbe positive. We have conducted a literature search in an attempt to make a comparison with documented MAIX isolates and other studies examining genetic variability within MAC strains, 
(a)

(129)

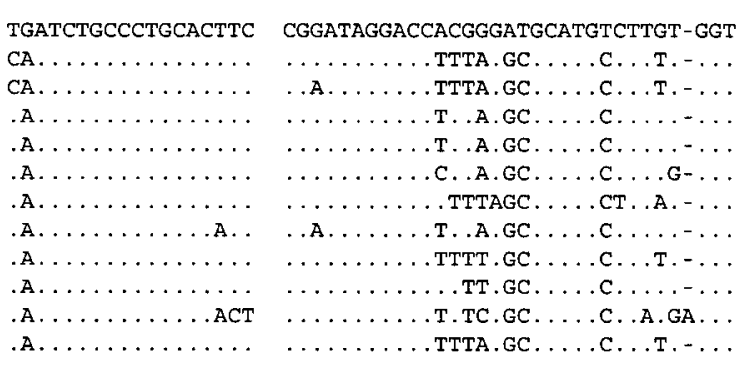

(248)

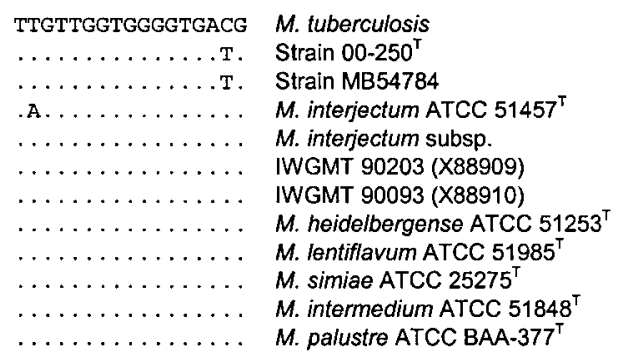

(b)

(644/249)

CAAGgGCGCCAAgGAgG

... TCG . . . . . . . .

. . TCG....... .

$\ldots$ TCG. . . . . . T.

. . . CAG. . . . . . . .

G. . TCG....... T.

. . . TCG. . . . . . .

$\ldots$ TCG.........

G. . TCG.......
(674/279)

(806/409)

GCAGATTGCGgCCACCGCAGCGATTTCGgCGGGTGACCAGTCCATCGGTGACCTG CTCGA M. tuberculosis (M15467)

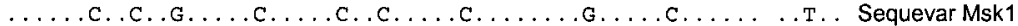

$\ldots \ldots$. .c. . .............................. Sequevar Msk2

$\ldots \ldots$..................................... Sequevar Msk3

$\ldots \ldots$. . . .....G.C. . ...C.......G............ Sequevar Msk4

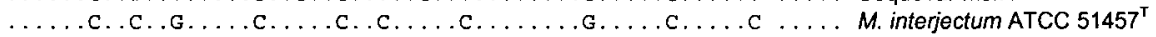

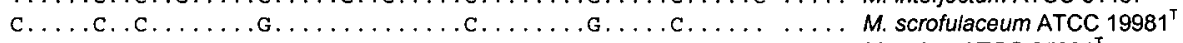

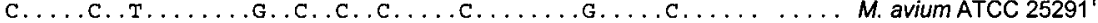

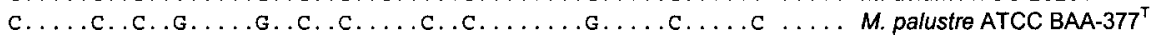

Fig. 3. (a) Alignment of hypervariable region $\mathrm{A}(\mathrm{V} 3)$ of the $16 \mathrm{~S}$ rRNA gene. Positions indicated are according to the $E$. coli sequence. In the regions shown, M. interjectum Patient 3 (AF014937) is identical to the type strain. 'M. interjectum subsp.' corresponds to sequences from GenBank designated $M$. interjectum Patient 1 (AF014935), M. interjectum Patient 2 (AF014936), M. interjectum MB739 (AJ272088) and IWGMT 90100 (X88915). All strains listed in the alignment have identical hypervariable regions $\mathrm{B}$, which are therefore not included. (b) Alignment of hypervariable regions of the partial $h s p 65$ gene. Numbers in parentheses above the alignment represent the position within the complete hsp65 gene in M. tuberculosis (M15467) (Shinnick, 1987) followed by the position within the amplified 441-bp fragment. All other sequences were determined in our laboratory.

which may or may not have been identified using DNA probes, to determine whether similar isolates have been described previously in addition to strain MCRO 8. The ITS1 sequences did not correspond to any of the 12 MAC ITS1 sequevars published previously (Frothingham \&

Table 3. PRA patterns of the closest relatives of the novel isolates by $h s p 65$ gene sequence

Fragments below $50 \mathrm{bp}$ have been omitted.

\begin{tabular}{|lcc|}
\hline Strain & BstEII & HaeIII \\
\hline Strain $00-250^{\mathrm{T}}$ (and 14 other strains) & 231,210 & 145,127 \\
Strain MB54784 & $231,131,79$ & 127,94 \\
M. interjectum ATCC $51457^{\mathrm{T}}$ & 231,210 & 145,127 \\
M. heidelbergense ATCC 51253 & 231,210 & 127,112 \\
M. lentiflavum ATCC $51985^{\mathrm{T}}$ & 441 & 145,127 \\
M. simiae ATCC $25275^{\mathrm{T}}$ & 231,210 & 185,127 \\
M. intermedium ATCC $51848^{\mathrm{T}}$ & 231,210 & $145,129,127$ \\
M. triplex ATCC $700071^{\mathrm{T}}$ & 325,116 & 145,127 \\
M. scrofulaceum ATCC $19981^{\mathrm{T}}$ & 231,210 & $145,127,95$ \\
M. avium ATCC $25291^{\mathrm{T}}$ & 231,210 & $127,103,59$ \\
M. palustre ATCC BAA-377 & 231,210 & 127,94 \\
\hline
\end{tabular}

Wilson, 1993), nor did they correspond to any ITS1 sequence in public databases.

Some studies based on PRA of the partial hsp65 gene have shown isolates with an identical profile to that determined for our sequevars Msk1, Msk2 and Msk3. We obtained sequence data from a study that included $h s p 65$ sequence data $(360 \mathrm{bp}$ ) for three strains that had an identical PRA profile to that of our strains (Smole et al., 2002) and compared these with our sequences. These three strains, M. avium v3 920A-120 (AF241202), M. avium v3 121B-60.1 (AF354282) and M. avium v3 953A-145 (AF354283), were found to differ from our sequevar Msk1 by 15, 15 and 19 mismatches, respectively. Another study describes isolates identified as M. avium III, with an identical PRA pattern, that hybridized exclusively with $M$. avium-specific probes and not M. intracellulare (Leao et al., 1999). It is unclear whether an MAC probe was done, and 16S rDNA data were not included. The proportion of M. avium III strains from human isolates was $6.5 \%$ (out of 107 isolates). A 441-bp hsp65 sequence available for strain M. avium III (AF126033) was found to be identical to that of M. avium v3 953A-145 and therefore distinct from our sequences, with $96 \cdot 4 \%$ sequence identity or 16 mismatches in the 441-bp fragment from Msk1. 
Another study suggested that the $M$. interjectum PRA pattern, which we determined to be identical to that of our strains, is also similar to M. simiae II-3 (Taylor et al., 1997), $M$. intracellulare 3 and M. intermedium I (da Silva Rocha et al., 2002). Our computer-generated analysis of $M$. intermedium shows an additional $129 \mathrm{bp}$ fragment in the HaeIII digest, which would be indistinguishable from the 127 bp fragment using gel electrophoresis. Further PRA comparisons with available data from PRA profiles alone were difficult due to subjectivity and procedure variations, particularly with gel concentrations (Brunello et al., 2001; Strässle et al., 2001), where discrepancies between sequencebased PRA pattern determination and published data from gel electrophoresis (Telenti et al., 1993) can reach up to 14 bases. While PRA is a common method of choice due to its ease and rapidity, the reliability of PRA pattern determination is somewhat limited and may occasionally result in misidentification (Strässle et al., 2001). Finally, even within what are determined to be isolates of the MAC, various PRA patterns are observed, some of which are similar to those of other NTM species.

We recognized a similarity between our isolates and strain FI-16795 (Tortoli et al., 1997) due its scotochromogenicity, MAC AccuProbe positivity and similar HPLC profile. This isolate was subsequently identified as $M$. palustre and was evaluated, along with other isolates of $M$. palustre, in the publication describing the species (Torkko et al., 2002).

\section{Clinical significance}

The patient described in this case history presented with a clinical history of bronchiectasis of unknown aetiology, documented first in around 1990 by CT. The absence of an alternative pathogenic agent supports the potential clinical relevance of this novel species. It is likely that this patient developed bronchiectasis from recurrent infections or an underlying lung disease, which rendered her susceptible to an atypical mycobacteriosis caused by this organism. It is well known that most NTM are not considered pathogenic; however, correct species determination is important in order to make appropriate treatment decisions. The limitations of biochemical testing were demonstrated in this case, as the NTM recovered initially from the patient was identified as M. gordonae and considered not to be responsible for the disease, even though the recovery of AFB from three sputum samples, according to the American Thoracic Society criteria (Wallace et al., 1997), would suggest a diagnosis of an NTM infection. Perhaps earlier intervention, in 1999, would have prevented further lung damage. All other isolates in our study were recovered from respiratory specimens, and no extensive clinical history is available. However, in two instances, the same isolate was recovered twice from the same patient in two different years. Also, one patient tested repeatedly from 1993 to 1996 yielded seven isolates, and the organism's pathogenicity in this case is therefore considered highly probable (Wallace et al., 1997).

\section{Differentiation of Mycobacterium saskatchewanense sp. nov. from other slowly growing NTM}

The group of unique strains evaluated in this study represent a species that, depending on the methodology used for identification, mimics various established species of mycobacteria. Without sequence-based identification, M. saskatchewanense sp. nov. may be difficult to recognize. Although the MAC AccuProbe is positive for these isolates, colony morphology and pigmentation are very distinct from $M$. avium or $M$. intracellulare. Additionally, the inability to grow at $42{ }^{\circ} \mathrm{C}$, a positive Tween 80 hydrolysis test and negative pyrazinamidase test differentiate this species from M. avium and M. intracellulare. M. saskatchewanense can also be differentiated from another recently described AccuProbe positive species, M. palustre, by its inability to grow at $42{ }^{\circ} \mathrm{C}$ and negative results for urease, pyrazinamidase and acid phosphatase. Biochemically, $M$. saskatchewanense most closely resembles $M$. gordonae, and phenotypic differentiation is subtle. This redundancy in biochemical profiles is not surprising, given the large number of mycobacteria currently established $(>100)$. The HPLC pattern of the strains evaluated in this study is distinct from the other late-emerging single-cluster peak patterns, with the exception of M. palustre, mainly in the relative heights of corresponding late peaks. The HPLC pattern is also very similar to some published profiles of strains identified as $M$. interjectum; however, it is distinct from the pattern observed in the type strain of $M$. interjectum. Despite sequence mismatches in the hsp65 gene, PRA analysis of $M$. saskatchewanense, $M$. interjectum, selected strains designated MAC and M. intermedium using enzymes BstEII and HaeIII revealed an identical pattern. While the type strain of $M$. intermedium is nonchromogenic, the morphologies of M. interjectum and $M$. saskatchewanense are similar. $M$. saskatchewanense can be differentiated from $M$. interjectum, MAC, M. gordonae, M. kubicae, M. palustre and other mycobacteria by its $16 \mathrm{~S}$ rRNA gene, ITS1 and $h s p 65$ gene sequences, confirming the isolate as representing a novel species.

\section{Description of Mycobacterium saskatchewanense sp. nov.}

Mycobacterium saskatchewanense (sas.kat.che.wa.nen'se. N.L. neut. adj. saskatchewanense pertaining to Saskatchewan).

Cells are acid-alcohol-fast, long, beaded rods. Grows at $25-37^{\circ} \mathrm{C}$, but not at $42{ }^{\circ} \mathrm{C}$. The organism shows a bright yellow pigment under both light and dark conditions. Strains with an identical $16 \mathrm{~S}$ rRNA gene sequence to that of $00-250^{\mathrm{T}}$ are MAC AccuProbe positive. Negative for acid phosphatase, arylsulfatase, $\beta$-glucosidase, niacin, nitrate reductase, pyrazinamidase and urease and does not grow on $5 \% \mathrm{NaCl}$. Some strains show a thin film of growth on MacConkey agar without crystal violet. Other variable test results include semi-quantitative catalase and tellurite reduction. Most isolates are strongly positive for Tween 80 
hydrolysis and all isolates have heat-stable catalase activity. Mycolic acids of the cell wall produce a single-cluster pattern by HPLC, typical of M. kubicae, M. palustre and some documented clinical strains of $M$. interjectum. Sequencing of the 16S rRNA, hsp65 gene and ITS1 region reveals unique data that are crucial in the differentiation of this species from selected NTM species. Isolates are susceptible in vitro to amikacin, clarithromycin, ciprofloxacin, linezolid, levofloxacin, moxifloxacin, rifabutin, rifampicin, streptomycin and sulfamethoxazole and are resistant to isoniazid. Eighty-six per cent of isolates are susceptible to ethambutol, with a resistance breakpoint of $10 \mu \mathrm{g} \mathrm{ml}^{-1}$. Strains have been isolated from human respiratory samples and were proven to be clinically significant in our case study. Strain MB54784 may constitute a distinct species, since it shows significant $h s p 65$ and ITS1 sequence variation; however, due to phenotypic and 16S rDNA similarities, this is difficult to establish at this time.

The type strain is strain $00-250^{\mathrm{T}}\left(=\mathrm{ATCC}\right.$ BAA $-544^{\mathrm{T}}=$ DSM $44616^{\mathrm{T}}=$ CIP $108114^{\mathrm{T}}$ ), which was isolated from the sputum of a patient with bronchiectasis.

\section{ACKNOWLEDGEMENTS}

We thank Professor Hans G. Trüper for his assistance in naming the species. We also thank Colleen Foster for her assistance.

\section{REFERENCES}

Brown, B. A., Wallace, R. J., Jr \& Onyi, G. O. (1992). Activities of clarithromycin against eight slowly growing species of nontuberculous mycobacteria, determined by using a broth microdilution MIC system. Antimicrob Agents Chemother 36, 1987-1990.

Brunello, F., Ligozzi, M., Cristelli, E., Bonora, S., Tortoli, E. \& Fontana, R. (2001). Identification of 54 mycobacterial species by PCR-restriction fragment length polymorphism analysis of the hsp65 gene. J Clin Microbiol 39, 2799-2806.

Butler, W. R. \& Guthertz, L. S. (2001). Mycolic acid analysis by high-performance liquid chromatography for identification of Mycobacterium species. Clin Microbiol Rev 14, 704-726.

Butler, W. R., Floyd, M. M., Silcox, V. A. \& 9 other authors (1996). Standardized Method for HPLC Identification of Mycobacteria. Atlanta: US Dept of Health and Human Services, Centers for Disease Control and Prevention.

Cregan, P., Yajko, D. M., Ng, V. L., Gonzalez, P. C., Nassos, P. S., Sanders, C. A. \& Hadley, W. K. (1992). Use of DNA probes to detect Mycobacterium intracellulare and " $\mathrm{X}$ " mycobacteria among clinical isolates of Mycobacterium avium complex. J Infect Dis 166, 191-194.

da Silva Rocha, A., Werneck Barreto, A. M., Dias Campos, C. E., Villas-Boas da Silva, M., Fonseca, L., Saad, M. H., Degrave, W. M. \& Suffys, P. N. (2002). Novel allelic variants of mycobacteria isolated in Brazil as determined by PCR-restriction enzyme analysis of $h s p 65$. J Clin Microbiol 40, 4191-4196.

Frothingham, R. \& Wilson, K. H. (1993). Sequence-based differentiation of strains in the Mycobacterium avium complex. J Bacteriol 175, $2818-2825$
Harmsen, D., Rothganger, J., Frosch, M. \& Albert, J. (2002). RIDOM: ribosomal differentiation of medical micro-organisms database. Nucleic Acids Res 30, 416-417.

Heifets, L., Lindholm-Levy, P., Libonati, J. P., Hooper, N., Laszlo, A., Cynamon, M. \& Siddiqi, S. (1993). Radiometric Broth Macrodilution Method for the Determination of Minimal Inhibitory Concentrations (MIC) with Mycobacterium avium Complex Isolates. Denver: National Jewish Center for Immunology and Respiratory Medicine.

Kent, P. T. \& Kubica, G. P. (1985). Public Health Mycobacteriology: a Guide for the Level III Laboratory. Atlanta: US Department of Health and Human Services, Centers for Disease Control.

Leao, S. C., Briones, M. R., Sircili, M. P., Balian, S. C., Mores, N. \& Ferreira-Neto, J. S. (1999). Identification of two novel Mycobacterium avium allelic variants in pig and human isolates from Brazil by PCR-restriction enzyme analysis. J Clin Microbiol 37, 2592-2597.

Lumb, R., Goodwin, A., Ratcliff, R., Stapledon, R., Holland, A. \& Bastian, I. (1997). Phenotypic and molecular characterization of three clinical isolates of Mycobacterium interjectum. J Clin Microbiol 35, 2782-2785.

NCCLS (2000). Susceptibility testing of mycobacteria, nocardia, and other aerobic actinomycetes. Tentative standard M24-T2, 2nd edn. Wayne, PA: National Committee for Laboratory Standards.

Rogall, T., Wolters, J., Flohr, T. \& Böttger, E. C. (1990). Towards a phylogeny and definition of species at the molecular level within the genus Mycobacterium. Int J Syst Bacteriol 40, 323-330.

Shinnick, T. M. (1987). The 65-kilodalton antigen of Mycobacterium tuberculosis. J Bacteriol 169, 1080-1088.

Smole, S. C., McAleese, F., Ngampasutadol, J., von Reyn, C. F. \& Arbeit, R. D. (2002). Clinical and epidemiological correlates of genotypes within the Mycobacterium avium complex defined by restriction and sequence analysis of hsp65. J Clin Microbiol 40, 3374-3380.

Soini, H., Bottger, E. C. \& Viljanen, M. K. (1994). Identification of mycobacteria by PCR-based sequence determination of the 32kilodalton protein gene. J Clin Microbiol 32, 2944-2947.

Soini, H., Eerola, E. \& Viljanen, M. K. (1996). Genetic diversity among Mycobacterium avium complex AccuProbe-positive isolates. J Clin Microbiol 34, 55-57.

Springer, B., Kirschner, P., Rost-Meyer, G., Schroder, K. H., Kroppenstedt, R. M. \& Bottger, E. C. (1993). Mycobacterium interjectum, a new species isolated from a patient with chronic lymphadenitis. J Clin Microbiol 31, 3083-3089.

Springer, B., Stockman, L., Teschner, K., Roberts, G. D. \& Bottger, E. C. (1996). Two-laboratory collaborative study on identification of mycobacteria: molecular versus phenotypic methods. J Clin Microbiol 34, 296-303.

Strässle, A., Truong, M. \& Pfyffer, G. E. (2001). Identification of non-tuberculous mycobacteria by PCR/restriction enzyme analysis of the $65-\mathrm{kDa}$ heat shock protein gene: trick or treat? In Abstracts of the 102nd General Meeting of the American Society for Microbiology, abstract U11. Washington, DC: American Society for Microbiology.

Taylor, T. B., Patterson, C., Hale, Y. \& Safranek, W. W. (1997). Routine use of PCR-restriction fragment length polymorphism analysis for identification of mycobacteria growing in liquid media. J Clin Microbiol 35, 79-85.

Telenti, A., Marchesi, F., Balz, M., Bally, F., Bottger, E. C. \& Bodmer, T. (1993). Rapid identification of mycobacteria to the species level by polymerase chain reaction and restriction enzyme analysis. J Clin Microbiol 31, 175-178.

Torkko, P., Suomalainen, S., livanainen, E., Tortoli, E., Suutari, M., Seppänen, J., Paulin, L. \& Katila, M.-L. (2002). Mycobacterium palustre sp. nov., a potentially pathogenic, slowly growing 
mycobacterium isolated from clinical and veterinary specimens and from Finnish stream waters. Int J Syst Evol Microbiol 52, 1519-1525.

Tortoli, E., Bartoloni, A., Burrini, C., Colombrita, D., Mantella, A., Pinsi, G., Simonetti, M. T., Swierczynski, G. \& Bottger, E. C. (1996). Characterization of an isolate of the newly described species Mycobacterium interjectum. Zentbl Bakteriol 283, 286-294.

Tortoli, E., Piersimoni, C., Kirschner, P. \& 10 other authors (1997). Characterization of mycobacterial isolates phylogenetically related to, but different from Mycobacterium simiae. J Clin Microbiol 35, 697-702.

Turenne, C. Y., Tschetter, L., Wolfe, J. \& Kabani, A. (2001). Necessity of quality-controlled $16 \mathrm{~S}$ rRNA gene sequence databases: identifying nontuberculous Mycobacterium species. J Clin Microbiol 39, 3637-3648.

Turenne, C., Chedore, P., Wolfe, J., Jamieson, F., Broukhanski, G., May, K. \& Kabani, A. (2002). Mycobacterium lacus sp. nov., a novel slowly growing, non-chromogenic clinical isolate. Int J Syst Evol Microbiol 52, 2135-2140.

Viljanen, M. K., Olkkonen, L. \& Katila, M. L. (1993). Conventional identification characteristics, mycolate and fatty acid composition, and clinical significance of MAIX AccuProbe-positive isolates of Mycobacterium avium complex. J Clin Microbiol 31, 1376-1378.

Wallace, R. J., Jr, Nash, D. R., Steele, L. C. \& Steingrube, V. (1986). Susceptibility testing of slowly growing mycobacteria by a microdilution MIC method with 7H9 broth. J Clin Microbiol 24, 976-981.

Wallace, R. J., Jr, Glassroth, J., Griffith, D. E., Olivier, K. N., Cook, J. L. \& Gordin, F. (1997). Diagnosis and treatment of disease caused by nontuberculous mycobacteria. Official statement of the American Thoracic Society. Am J Respir Crit Care Med 156, S1-S25.

Wallace, R. J., Jr, Brown-Elliott, B. A., Ward, S. C., Crist, C. J., Mann, L. B. \& Wilson, R. W. (2001). Activities of linezolid against rapidly growing mycobacteria. Antimicrob Agents Chemother 45, 764-767. 